\title{
Feasibility and Optimality of Sustainable Growth under Materials
}

\author{
Balance \\ Ken-Ichi Akao* and Shunsuke Managi** \\ *School of Social Sciences, Waseda University \\ ** Faculty of Business Administration, Yokohama National University
}

August 9, 2006

\begin{abstract}
Pollution from consumption and production is an inevitable part of economic processes. We employ a materials balance approach and develop an endogenous growth theory, with recycling activity, to examine the evolution of the economic and environment systems. This paper provides feasibility and optimality conditions for sustainable economic growth with rising environmental quality. The fundamental condition of feasibility is that the flow of natural resources, which eventually returns to the environment as waste, has a negative growth rate in the long run. Recycling may not necessarily matter for an optimal sustainable growth.
\end{abstract}

J.E.L. Classification Code O41, Q01, Q53, Q56.

\footnotetext{
* Corresponding author. School of Social Sciences, Waseda University, 1-6-1 Nishiwaseda Shijuku, Tokyo 169-8050 Japan, Tel: 81-3-5286-1908 / Fax: 81-3-3204-8962. E-mail address: akao@waseda.jp (Ken-Ichi Akao).

We are grateful to two anonymous referees, K. Domon, Y.H. Farzin, H. Koide, S. Nakamura, and A. Onuma for detailed comments that substantially improved the paper. Partial financial support from Waseda University (1999A24-2) and the Ministry of Education, Science, Sports, and Culture of Japan (11730017) are gratefully acknowledged.
} 


\section{Introduction}

Rapid economic growth tends to be detrimental to the environment due to a greater use of natural resources and higher levels of pollutant emissions. Hence, arises the issue of a potential conflict between economic policies and environmental qualities.

Economists are interested in technology and preferences by human beings as fundamentals for analyzing long-run economic growth. In recent literature on economic growth and the environment, environment's ability to dissipate waste has been added to the analysis (Brock and Taylor, 2006). Considering the environment as a long-term consequence of the economy, however, is more than merely adding a new factor. That is, it is necessary to investigate a holistic economic and environment system instead of a relatively small economic subsystem. Essential to this approach is consideration of the law of technology which has as a basis both human beings and nature. The fundamental law of conservation of mass or the mass balance principle is an essential element of this law. This point has been somewhat slighted in much of the existing literature.

In the initial periods of environmental economics, the importance of the fundamental law of conservation of mass was raised by Ayres and Kneese (1969). They noted that, "the externalities associated with the disposal of residuals resulting from the consumption and production process must be viewed ... as ... [an] inevitable part of these [economic] processes. ... The common failure to recognize [their economic significance] may result from viewing the production and consumption processes in a manner that is somewhat at variance with the fundamental law of conservation of mass" (Ayres and Kneese, 1969, 282-3). This study will take into account this law and the spirit of Ayres and Kneese (1969), and analyze the relationship between economic growth and environment. An additional benefit of considering this law is that we will be able to take into account a missing piece of the literature. 
The literature on economic growth and the environment mainly focused on the production side. Thus, from that we might conclude that economic growth could continue without bounds if increased productive capacity allowed for both consumption growth and improved environmental quality (Stokey, 1998; Aghion and Howitt, 1998). However, once we consider the waste from consumption, then environmental degradation increases as it grows. The environmental risks from an increasing volume of consumption more than outweigh improvements in resource efficiency on the production side.

Chapter 4 of Agenda 21 (United Nations, 1993) ${ }^{1}$ identifies that: "the major cause of continued deterioration of the global environment is the unsustainable patterns of consumption and production." Sustainable consumption and production are closely related to sustainable development. For example, the Johannesburg Summit on Sustainable Development World Summit in 2002 agreed that "changing consumption and production patterns is one of the overarching objectives of, and essential requirements for sustainable development."

We take into account the negative effects of both production and consumption by incorporating a materials balance approach into the endogenous growth theory. The flow of natural resources (materials) taken from the environment goes through various transformational processes, such as production, consumption, recycling, and is ultimately returned to the environment (see Ayres 1999). The law of conservation of mass states that weight is invariant over any transformation process and place. This, thus, consistently captures the interaction between the two systems of the economy and the environment. Conventional economic analyses, including environmental economics, however, do

\footnotetext{
${ }^{1}$ Agenda 21 was adopted by more than 178 Governments at the United Nations Conference on Environment and Development (UNCED) held in Rio de Janeiro, Brazil, 3 to 14 June 1992. It is a comprehensive plan of action to be taken globally, nationally and locally by organizations of the United Nations System, Governments, and Major Groups in every area in which human impacts on the environment.
} 
not fully account for the basic physical law of mass conservation though always dealing with various aspects of the materials flow. In particular, these are missing in the literature on economic growth and the environment.

Our goal in this paper is to provide feasibility and optimality conditions for long run economic growth with rising environmental quality under a materials balance. Our model investigates pollution from both the production and consumption sides.

This paper is structured as follows. Section 2 provides background. Section 3 investigates the feasibility of sustainable growth. Section 4 models the optimal sustainable growth. Section 5 presents a summary and concluding remarks.

\section{Background}

The Brundtland report's Our Common Future (WCED, 1987) defines sustainable development as "development that meets the needs of the present without compromising the ability of future generations to meet their own needs." This definition is ambiguous and raises more questions than it answers (Heal, 1998). A more precise definition would, for example, require utility levels, or resource stocks, or total capital stocks including natural capital and human capital to be non-decreasing over time (Pezzey, 1992 ; Smulders, 2000; Asheim et al., 2001). Thus, sustainable paths would confront standard optimal solutions as formalized in the traditional theory of economic growth (Pezzey, 1997).

Endogenous growth theory has been used to analyze economic growth and the environment (e.g., Stokey, 1998; Aghion and Howitt, 1998; Jones and Manuelli, 2001; Andreoni and Levinson, 2001; Xepapadeas, 2003; Hartman and Kwon, 2005; Brock and Taylor, 2006). Stokey (1998) and Aghion and Howitt (1998, Chapter 5) obtain a set of necessary conditions under which it is optimal to sustain both economic growth and environment conservation. One of their conditions is that the engine of 
economic growth should be in an industrial sector that does not cause environmental degradation ${ }^{2}$. An intuitive explanation is as follows: Along a sustainable growth path, optimal pollution regulations become stricter. This increases the environmental expenditure of the regulated industry and, thus, lowers its net marginal productivity of capital. Economic growth ceases when this marginal productivity declines to the level of the discount rate of a representative household. However, if the economy is supported by a clean industry that is a growth engine, the regulated industry can keep productivity higher than the discount rate by increasing input from the clean industry. As a result, the economy sustains growth on an optimal path, accompanied by environmental conservation.

It is plausible to think that long-term economic growth can be sustainable by the accumulation of human capital and knowledge, which generally do not damage the environment. Therefore, this scenario is very appealing to those who wish for sustainable development. In this manner, only pollution from production is analyzed, and pollution associated with the disposal of residuals from consumption is not considered although it is common in the literature on growth and the environment. Research attention should be paid to the consumption side as well. As we shall see, when we incorporate residuals into a growth and environment model, sustainable growth no longer becomes feasible under a certain assumption. For the study of growth and the environment, this suggests the need for a more comprehensive approach to capture the system of the economy and environment. To this purpose, we employ a materials balance approach.

Conventional environmental economic analysis dealt with aspects of the materials flow without taking into full account the basic physical law of mass conservation. One exception is in a paper

\footnotetext{
${ }^{2}$ The engine for economic growth is an industrial sector whose product grows rapidly enough to support sustainable final goods production along an optimal path. The sector is R\&D which causes Schumpeter's creative destruction in the models of Aghion and Howitt (1998), and is referred to as exogenous technological progress in the models of Stokey
} (1998). 
by Ayres and Kneese (1969). Using the law of conservation of mass, they study the residualsgenerating materials flow of production and consumption with its link to pollution. They argued that in economic modelling the problem of environmental degradation could not be adequately assessed unless the complete economic materials flow was considered along with the law of conservation of mass and its corollary, the mass balance principle (MBP). The MBP, a direct consequence of the first law of thermodynamics, states that the mass of input, including any un-priced material from the environment, must exactly equal the mass of output including waste at each physical transformation. Thus, the entire materials flow is at the core of environmental degradation. Ayres and Kneese (1969) employ a static general equilibrium model with linear technology. In their model, residuals are generated with rigid joint production. The amount of a residual can be reduced if and only if goods output is reduced along with raw materials and services involved. Pethig (2005) extends this model to allow pollution abatement activities as well as nonlinear technology. Recycling activities play an important role in protecting the environment (Smith, 1972; Eichner and Pethig, 2001). The relationship between recycling and economic growth has been studied by Di Vita (2001) and Pittel et al. (2005). In particular, Pittel et al. (2005) adopt a convincing formulation for a materials balance, which is similar to our formulation, although their interest is somewhat different from this study. In the context of sustainable development, van den Bergh and Nijkamp (1994) and Ayres and van den Bergh (2005) study macro dynamic models incorporating MBP and show simulation results. Their models employ specific functional forms and parameters and lack the micro foundation, with which the present study differs. 


\section{Feasibility of Sustainable Growth}

\subsection{Impossibility result of economic growth}

The model employed here is an extension of the traditional growth model with a materials balance approach. The materials balance approach imposes a MBP on the entire flow of materials where the total weight of materials is the same at all points along the flow. In this environment-economic model, all material resources extracted from the environment eventually end up as residuals in the environment.

To describe the economy, we use a simple macroeconomic equation:

$$
\dot{K}=Y-v(C+\delta K), \delta>0, v \in(0,1]
$$

where $Y, C$, and $K$ stand for aggregate output, consumption, and capital stock, respectively ${ }^{3}$. The positive constant $\delta$ is the depreciation rate of the capital. Consumption and scrapped capital $C+\delta K$ constitute primal waste. Part of primal waste $(1-v)(C+\delta K)$ is recycled, so that the net waste is equal to $v(C+\delta K)$. We refer to $(1-v)$ as the recycling rate. Recycled goods $(1-v)(C+\delta K)$ should be consumed or invested. In this continuous time model, we assume that all recycled goods are invested. This assumption expresses the time lag between the production and the consumption of recycled goods.

The economy exploits the environment in two forms: as a source of natural resources and as a sink of waste. As a dichotomy we call the former the unpolluted environment and the latter the polluted environment. Let $M$ and $D$ denote the stocks of the unpolluted and polluted environment,

\footnotetext{
${ }^{3}$ Throughout the paper, we use the following notation for function of time $x:[0, \infty) \rightarrow(-\infty, \infty): \dot{x}:=d x / d t$ (the time derivative); $g_{x}:=\dot{x} / x$ (the growth rate); $x_{0}:=x(0)$ (the initial value).
} 
respectively. Assume their evolutions are described as

$$
\begin{aligned}
& \dot{M}=-R+\theta D, \theta>0 \text { (constant), } \\
& \dot{D}=P-\theta D, \quad D \in\left(0, D_{\max }\right),
\end{aligned}
$$

where $R \geq 0$ is the flow of natural resources, $\theta>0$ is the coefficient which expresses the ability for natural assimilation, and $P \geq 0$ is pollution flow. ${ }^{4}$ The net flow of pollution adds to the stock of a polluted environment, and a certain part of this stock is broken down in each period due to the assimilative capacity of the environment. While the constant exponential pollution decay (constant $\theta$ ) is assumed quite commonly in the literature of growth and the environment, it has been criticized for its unrealistic stance that the more environment is polluted, the greater are its assimilative capacities (Chevé, 2000). We treat this problem by setting the upper bound $D_{\max }$ to the stock of the polluted environment. $D_{\max }$ is a critical level at which once the environment is degraded, it completely loses its assimilative capacity and the economy inevitably suffers fatal damage. This assumption is also used by Aghion and Howitt (1998).

Since we aim at capturing the whole system of the economy and environment, the environment described by (2) and (3) may correspond to the geosystem, which consists of the global cycles of water, carbon, nitrogen and other primal materials on the earth. In this context, we could consider $R, M$ and $D$ as the elements of the global carbon cycle. For example, the $R$ is the carbon flow in the forms of oil, charcoal, timber, and grain among others. The $M$ is the useful carbon stock such as fossil fuels in the ground, forest stock, grain field and populations of fish. The $D$ is harmful carbon stock

\footnotetext{
${ }^{4}$ With respect to several environmental problems, accumulated pollution on ecological systems is more realistic and adds interesting transition dynamics to materials flow analysis rather than just the flow effects of pollution. Stock pollution is analyzed, for example, in Bovenberg and Smulders (1995) and Aghion and Howitt (1998).
} 
such as the atmospheric carbon and toxic organic compounds including VOCs and POPs. Under this interpretation, fossil fuels and forests are alike as storages of carbon. So, in this paper we do not distinguish between renewable and nonrenewable resources. However, speed of natural regeneration (or assimilation) differs among environmental assets. This difference cannot be expressed in our model since the environment is aggregated with a single state variable. Instead, we could interpret that the magnitude of $\theta$ reflects the composition of natural resources. A very low regeneration rate implies that the economy heavily depends on nonrenewable resources. The magnitude of $\theta$ could also reflect the composition of pollutants. That is, low assimilation rate implies that the major pollution emissions and effluents are difficult to decompose. We will see how $\theta$ affects the feasibility and optimality of sustainable growth.

Using this setup, we show that steady state growth is impossible under a certain connection between the economy and the environment. Borrowing the terminology of Barro and Sala-i-Martin (1995, Chapter 1), we call the state of the economy steady state growth, if the variables on the right side of (1) satisfy the following conditions: $:^{5}$ (a) All growth rates $\left(g_{Y}, g_{C}, g_{K}\right.$, and $\left.g_{v}\right)$ are constant. (b) $g_{Y}, g_{C}$, and $g_{K}$ are non-negative and at least one of them is positive. (c) $g_{v} \leq 0 .{ }^{6}$ Lemma 1 below characterizes a steady state growth path. Lemma 1 also states an interesting result for recycling activities: on a steady state growth path, recycling rates should be constant.

Lemma 1. Steady state growth implies $g_{Y}=g_{K}=g_{C}>0$ and $g_{v}=0$. That is, steady state growth is balanced growth, and along such a path, recycling rates are constant. Sustained improvement of recycling rates $\left(g_{v}<0\right)$ is not compatible with steady state growth: $g_{v}<0$ is accompanied by $g_{K}<0$ and/or $g_{Y}<0$.

\footnotetext{
${ }^{5}$ Since we are interested in the long-term consequences of economic growth, we consider only a constant growth rate for each variable, assuming it represents the long-term average growth rate for the variable.

${ }^{6}$ If $g_{r}>0$, the upper bound of $r$ is violated.
} 
Proof. See the Appendix.

An impossibility result is obtained by assuming that all waste damages the environment, i.e.

$$
P=v(C+\delta K) .
$$

Then, (3) is rewritten as

$$
\dot{D}=v(C+\delta K)-\theta D .
$$

When the economy is on a steady state growth path, Lemma 1 implies

$$
v(t)(C(t)+\delta K(t))=v_{0}\left(C_{0}+\delta K_{0}\right) e^{g t},
$$

where $g$ is the balanced growth rate $\left(g=g_{Y}=g_{K}=g_{C}\right)$. If $g>0, P=v(C+\delta K)$ grows unfettered. It is easy to see that the stock of polluted environment $D(t)$ reaches the critical level $D_{\max }$ within a finite time. Therefore, steady state growth leads the economy to an environmental catastrophe. Notice that this holds irrespective of the magnitude of the growth rate $g$, the recycling rate $1-v$, the assimilation ability $\theta$, the initial value of the polluted environment $D_{0}$, and the critical level $D_{\max }$. Therefore, we have:

Proposition 1. Suppose that the evolution of the environment is described by (5). Even in an economy where there is active recycling $(v<1)$, any steady state growth is impossible.

The literature on growth and environment usually deals with harmful effects on the environment by the production sector. Pollution abatement technology plays an important role to have both economic growth and pollution reduction. Departing from this tradition, we deal with the production sector and the consumption sector, since we intend to understand the effects on the environment 
in a more comprehensive manner. The above impossibility result is obtained by incorporating the consumption sector into the model, which brings the novel result. That is, there is waste from the consumption sector which is not by-product of consumption, but transformation of consumption goods. Such waste cannot be reduced by pollution abatement activities, but by recycling. In order to reduce environmental degradation due to the waste, recycling rate must go up faster than the growth rate of consumption so that $g+g_{v} \leq 0$, since (5) implies

$$
D(t)=\frac{v_{0}\left(C_{0}+\delta K_{0}\right)}{g+g_{v}+\theta} e^{\left(g+g_{v}\right) t}+\left(D_{0}-\frac{v_{0}\left(C_{0}+\delta K_{0}\right)}{g+g_{v}+\theta}\right) e^{-\theta t}
$$

But, Lemma 1 shows that on a steady state growth path, the recycling rate is constant $\left(g_{v}=0\right)$. Therefore, in contrast to existing literature, it is impossible to have both economic growth (increase of consumption) and environmental conservation. The waste problem imposes a limit to growth. In Proposition 1, we also take into account the waste from production sector with assumption of a fixed depreciation rate. By the same logic, the waste also imposes a limit to growth, although it is additional and unlike the consumption sector, the waste may be reduced by choice of technology.

\subsection{Materials balance approach}

In the previous section, we assumed that all waste eventually becomes pollution flow. However, we need not consider that all waste actually harms the environment. In endogenous growth theories, sustainable economic growth is enabled by the accumulation of intangible knowledge and technology (Aghion and Howitt, 1998). Following this idea, we consider waste as a combination of the tangible and the intangible. The intangible does not damage the environment.

As a more elaborate description for the connection between the environment and the economy than (4), this study applies a materials balance approach. ${ }^{7}$ The notion of weight can be introduced in

\footnotetext{
${ }^{7}$ As mentioned in the introduction, the pioneer and seminal paper adapting materials balance approach is Ayres
} 
order to distinguish between tangible and intangible waste. Tangible waste has weight and becomes pollution flow. The origin of tangible waste is material extracted from nature. Let the flow of natural resources $R(t)$ be measured by weight. We assume that the production process causes the natural resource flow into the final goods. In general, weight of the production equals natural resource flow as input and a part of capital stock used in the process. In this preliminary study of MBP, we simply assume the weight of production equated to the natural resource flows into the final goods. One simple example is that material is brought into a factory and processed into final goods. The factory is considered as capital but not included in the final goods. It follows that the weight of output $Y(t)$ is equal to $R(t)$. The tangible waste from consumption $C(t)$ is expressed by the weight unit as

$$
v(t) C(t) \frac{R(t)}{Y(t)}
$$

where the value of $(R / Y)$ is used to transfer the consumption to weight unit. Let $w_{K}(t)$ be the weight of one unit of capital and $W(t)$ be the weight of the whole capital at $t .{ }^{8}$ From identity $W(t)=w_{K}(t) K(t)$, the tangible waste from scrapped capital $v(t) \delta K(t)$ is expressed as

$$
w_{K}(t) v(t) \delta K(t)=v(t) \delta W(t) .
$$

We assume that the pollution flow $P(t)$ is equivalent to the tangible waste that is discharged into the environment. The waste is measured by weight and comes from both the production and consumption sectors. Then, the sum of the two types of tangible waste becomes the pollution flow:

$$
P(t)=v(t)\left(C(t) \frac{R(t)}{Y(t)}+\delta W(t)\right) .
$$

and Kneese (1969). See also Smith (1972), Mäler (1974), Pethig (2005) and Pittel et al. (2005).

${ }^{8} W(t)$ is also referred to as the weight of the ecomomy. 
We thus have the state equation for the polluted environment as follows:

$$
\dot{D}(t)=v(t)\left(C(t) \frac{R(t)}{Y(t)}+\delta W(t)\right)-\theta D(t), \quad D(t) \in\left(0, D_{\max }\right)
$$

There is a physical constraint imposed by the law of conservation of mass: The total weight of the economy and the environment is constant over time. Recall that the environment is divided into polluted and unpolluted sections. The polluted part $D$ and the unpolluted part $M$ are measured by weight. Then, the law of conservation of mass implies

$$
W+(M+D)=\text { constant }
$$

where $(M+D)$ is the weight of the environment, while $W$ is the weight of the economy. Differentiate (9) with respect to time and substitute (4) and (5). Then, we have the state equation for the weight of the economy:

$$
\dot{W}=-\dot{M}-\dot{D}=-P+R,
$$

or substituting (7),

$$
\dot{W}=-v\left(R \frac{C}{Y}+\delta W\right)+R
$$

with which the system of the economy and the environment is closed in terms of a materials balance. See Figure 1 for a conceptual relationship of the materials balance in this study.

\section{$<$ Figure 1 $>$}

Independent of our materials balance approach, Pittel et al. (2005) derive a similar formulation. There are two distinctive features in our formulation. First, our formulation covers the whole system of the economy and environment and captures the circulation of materials between the economy and 
the environment, using a few simple equations without specifying functional forms for production technology. Therefore, the results are rather general and could have broad application. Second, we explicitly use two types of units for the economic and environmental variables. One is the usual economic unit, which is used to show the functional relationship of the variables through functions such as utility and production functions. The other is the weight unit, which is used to track materials (tangible goods) and to express materials balance. These two units enable us to distinguish tangible and intangible goods and to discuss the relationship between sustainable growth and dematerialization.

To make the latter feature clear, consider capital accumulation. We have two expressions of capital stock at $t: K(t)$ in the economic unit and $W(t)$ in the weight unit. Let $I(s, \tau)$ be the amount of capital stock of vintage $\tau$ at time $s$. In other words, it is the amount of capital installed at $\tau$ and remaining at $s$. Let $I(s, \tau)$ be measured by the economic unit. Then, we can express the capital stock at $t$ as

$$
K(t)=\int_{-\infty}^{t} I(t, \tau) d \tau
$$

in the economic unit. Since $I(s, \tau)$ is the amount of capital installed at $\tau$, the unit weight is the same as the product at $\tau, Y(\tau)$. Therefore, the weight expression of the capital stock is

$$
W(t)=\int_{-\infty}^{t} \frac{R(\tau)}{Y(\tau)} I(t, \tau) d \tau
$$

where $R(\tau) / Y(\tau)$ has the unit of weight. ${ }^{9}$ In order to verify our two unit approach, we show that these equations are equivalent to the associated state equations (1) and (10), respectively, with

\footnotetext{
${ }^{9}$ This vintage expression is owed to a referee of this journal.
} 
appropriate initial values. Differentiate these equations with respect to time and we have

$$
\begin{aligned}
\dot{K}(t) & =I(t, t)+\int_{-\infty}^{t} \frac{\partial I(t, \tau)}{\partial s} d \tau \\
\dot{W}(t) & =\frac{R(t)}{Y(t)} I(t, t)+\int_{-\infty}^{t} \frac{R(\tau)}{Y(\tau)} \frac{\partial I(t, \tau)}{\partial s} d \tau .
\end{aligned}
$$

$I(t, t)$ on the right hand side of each equation is the new investment at $t$, where "new" means that it is part of the new product at $t, Y(t)$. It has the same unit weight as $Y(t)$ and $C(t)$ and satisfies the following equation:

$$
I(t, t)=Y(t)-C(t)+(1-v) C(t)=Y(t)-v C(t) .
$$

On the other hand, there is old investment, where "old" means that it is recycled from scrapped vintage capital. The old investment differs from the new investment in the unit weight. Since capital stock depreciates exponentially with rate $v \delta$, we have $I(t, \tau)=I(\tau, \tau) e^{-v \delta(t-\tau)}$. Therefore,

$$
\frac{\partial I(t, \tau)}{\partial s}=-v \delta I(t, \tau)
$$

From this, we have

$$
\int_{-\infty}^{t} \frac{\partial I(t, \tau)}{\partial s} d \tau=-v \delta K(t) \text { and } \int_{-\infty}^{t} \frac{R(\tau)}{Y(\tau)} \frac{\partial I(t, \tau)}{\partial s} d \tau=-v \delta W(t)
$$

Notice that these show the net old investment, i.e. the scrapped capital (negative value) plus the old investment $(-\delta K(t)+(1-v) \delta K(t)$ in the economic unit). 
Substitute these results to have (1) and (10):

$$
\begin{aligned}
\dot{K}(t) & =[Y(t)-v C(t)]-v \delta K(t)=Y(t)-v(C(t)+\delta K(t)) \\
\dot{W}(t) & =\frac{R(t)}{Y(t)}[Y(t)-v C(t)]-v \delta W(t)=-v\left(R(t) \frac{C(t)}{Y(t)}+\delta W(t)\right)+R(t) .
\end{aligned}
$$

While capital stock in the economic unit $K(t)$ is a mix of tangible and intangible capitals, its weight $W(t)$ expresses the amount of tangible part of the capital stock. Notice that if $W(t)$ is proportional to $K(t)$ (in other word, if the unit weight of capital stock $w_{K}(t)=W(t) / K(t)$ is constant over time), then steady state growth is impossible by the same reason as in Proposition 1. However, $W(t)$ and $K(t)$ have different evolution equations. Therefore, it may be possible that while $K(t)$ grows, $W(t)$ grows less rapidly or decreases. This is dematerialization. Without dematerialization, steady state growth under environmental constraints is impossible.

\subsection{Possibility result of economic growth}

The environment-economy connection under materials balance is now described with (1), (8) and (10). In this section, we show impossibility and possibility results of steady state growth in this framework. Since there has been no attempt to investigate the feasibility of sustainable growth under materials balance, the following propositions are the first results on it, as far as the authors know.

First, we show an impossibility result. It is conditional unlike the case of Proposition 1.

Proposition 2. Consider an economy where the pollution flow is defined by (7). If the growth rate of natural resource flow $g_{R}$ is a positive and constant, any steady state growth reaches an environmental catastrophe $D_{\max }$ within a finite time. Therefore, steady state growth is impossible. ${ }^{10}$

\footnotetext{
${ }^{10}$ This impossibility result can be extended to the case where $g_{R}$ is not necessarily constant but satisfies
} 
Proof. Assume that the assertion is false, i.e., $\sup _{t \geq 0} D(t) \leq D_{\max }$. By Lemma 1, a steady state growth path has a constant recycling rate $1-v \in[0,1)$. Therefore, on a steady state growth path, (10) is solved as

$$
W(t)=\left(W_{0}-\frac{R_{0}\left(1-v C_{0} / Y_{0}\right)}{g_{R}+v \delta}\right) e^{-v \delta t}+\frac{R_{0}\left(1-v C_{0} / Y_{0}\right)}{g_{R}+v \delta} e^{g_{R} t}
$$

Note

$$
1-v C_{0} / Y_{0}=\left(g_{K}+v \delta\right) K / Y(>0)
$$

from (1). $g_{R}>0$ implies that there is $T \geq 0$ such that for all $t \geq T, W(t) \geq \theta D_{\max } /(v \delta)$ and thus, $\dot{D}(t)>v e^{g_{R} t} R_{0} C_{0} / Y_{0}>0$. This implies $\lim _{t \rightarrow \infty} D(t)=\infty$, a contradiction.

Proposition 2 shows that in order to realize steady state growth, the economy needs not only dematerialization mentioned above, but also the reduction of dependence on natural resources $\left(g_{R} \leq\right.$ $0)$.

For the case with $g_{R} \leq 0$, we can construct a steady state growth path with environmental conservation. We call the state of the economy sustainable growth if it has a steady state growth and if over time the environment is never degraded $\left(g_{D} \leq 0\right)$.

Assume the growth rates of $W$ and $D$ are constant. Then, a sustainable growth path is characterized in what follows. From (10),

$$
g_{W}=-v \delta+(R / W)(1-v C / Y) \cdot(\text { constant })
$$

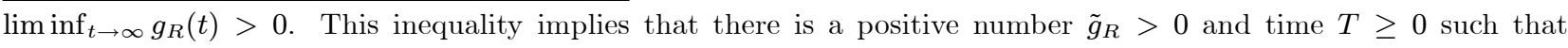
$g_{R}(t) \geq \tilde{g}_{R}$ for all $t \geq T$. 
By (12) in the proof of Proposition $2,1-v C / Y \neq 0$. Therefore, $g_{R}=g_{W}$. Then, from (8),

$$
g_{D}=v\left(\frac{C}{Y}+\delta \frac{W}{R}\right) \frac{R}{D}-\theta, \text { (constant) }
$$

and we thus have $g_{R}=g_{D}$. Also, it holds that $\dot{W}+\dot{D}=g_{R}(W+D)=R-\theta D$. The last equality follows from (8) and (10). Therefore, we have:

$$
g_{R}=g_{D}=g_{W}=\frac{R-\theta D}{W+D}
$$

Let

$$
\Omega=\frac{Y / K}{R / W}
$$

Notice that $R / W$ is the output/capital ratio in terms of the weight. Therefore, $\Omega$ is the transformation coefficient from the ratio in materials balance to the one in usual economic units, $Y / K$. Combine (12) and (13) to obtain

$$
g_{K}+v \delta=\Omega\left(g_{D}+v \delta\right)
$$

From (17) and Lemma 1, economic growth rates are characterized as

$$
g_{Y}=g_{K}=g_{C}=\Omega\left(g_{D}+v \delta\right)-v \delta .
$$

Notice that if $\Omega \leq 1, g_{K}>0$ and $g_{D} \leq 0$ are not compatible and thus sustainable growth is impossible. Keeping $\Omega>1$ implies that sustainable growth needs to maintain a high productivity of capital, in spite of the accumulation of capital stock, which implies that sustainable development needs an engine for economic growth. We will make further remark below Proposition 3. 
Finally, using (13), the consumption/output ratio on a sustainable growth path is obtained:

$$
\frac{C}{Y}=\frac{1}{v}\left[1-\left(g_{W}+v \delta\right) \frac{W}{R}\right]
$$

Notice that sign conditions are imposed on the variables characterized in (15), (18) and (19): $g_{R} \leq 0, g_{K}>0, C / Y>0$, which derives necessary and sufficient conditions for a sustainable growth to be feasible. Then, we have:

Proposition 3. Suppose that the economy and the environment are described by (1), (8) and (10).

(a) For given $K_{0}, W_{0}$, and $D_{0}$, there exists a sustainable growth path with constant growth rates for $R, W$, and $D$, if and only if the following four conditions are met:

1.

$$
g_{v}=0
$$

2.

$$
v<\theta D /(\delta W)
$$

3.

$$
\theta D \geq R>\max \left[R_{L}(v),-(W / D) R_{L}(v)\right]
$$

where $R_{L}(v)=\theta D-v \delta(W+D)$.

4. The coefficient $\Omega$ defined in (16) is sufficiently high to make the economic growth rate in (18) positive.

(b) The sustainable growth path is characterized by (15), (18) and (19).

Proof. Condition 1 (20) follows from Lemma 1. For Condition 3, the first inequality in (22), 
$\theta D \geq R$, is equivalent to $g_{R} \leq 0$ by (12). On a sustainable growth path, $g_{K}>0$ and $C / Y>0$ must hold. In the equations (18) and (19), this implies

$$
0<g_{W}+v \delta<R / W
$$

Substitute (15) and rearrange it to obtain a second inequality in $(22)$. Note that $R_{L}(v)$ and - $(W / D) R_{L}(v)$ have opposite signs. If $v \leq \theta D /[\delta(W+D)]$, then $R_{L}(v) \geq 0$, and there exists $R_{0}>0$ which satisfies $(22)$, because $\theta D_{0}>\theta D_{0}-v \delta\left(W_{0}+D_{0}\right) \geq 0$. On the other hand, if $v>\theta D /[\delta(W+D)]$, i.e., if $R_{L}(v)<0$, we need a condition to ensure the existence of $R_{0}>0$ such that $\theta D_{0}>-R_{L}(v)\left(W_{0} / D_{0}\right)$. This inequality is equivalent to $v<\theta D /(\delta W),(21)$ in Condition 2 . Note $\theta D /(\delta W)>\theta D /[\delta(W+D)]$. Finally, Condition 4 is necessary. Without it, $0<g_{W}+v \delta$ in (23) does not necessarily imply $g_{K}>0$.

This proposition has several implications:

(1) To realize sustainable growth, neither tangible input from nature nor tangible industrial waste must exceed its assimilation capability: $\theta D \geq \max [v \delta W, R]$. (This follows from (21) and (22)).

(2) Condition 2 shows that recycling activities are necessary if we choose a sustainable growth path with $\theta D /(\delta W)<1$. In other cases, sustainable growth is feasible without recycling activities. This implies that although a recycling activity may be necessary in a transition phase of the economy, it does not necessarily matter on a sustainable growth path.

(3) A rise of the recycling rate $(1-v)$ increases the consumption/output ratio, $C / Y$, meaning $\partial(C / Y) / \partial v<0$. (It follows from (19). The effect on the growth rate of the economy $g_{K}$ is ambiguous since

$$
\frac{\partial g_{K}}{\partial v}=-(C+\delta K)-v \frac{Y}{K} \frac{\partial(C / Y)}{\partial v}
$$


It does not affect environmental improvement $g_{D}$. It is natural resource flow $R$ that affects $g_{D}$ (see $(15))$.

(4) The increase of material flows $R$ slows the improvement of the environment (higher $g_{D}$ ) and enhances the economic growth (higher $g_{K}$ ). (The former result follows from (15) and the latter is obtained by differentiating (18) with respect to $R$.)

(5) The time path of natural resource flow must be nonincreasing $\left(g_{R} \leq 0\right)$. Note that for the case of $g_{R}<0$, we use natural resources as if they were nonrenewable.

(6) Finally, $g_{K}+v \delta=\Omega\left(g_{D}+v \delta\right)$ (17) is the fundamental equation for the relationship between the economy and the environment, showing the trade-off between economic growth and environment conservation. An interesting point is that sustainable growth requires $g_{K}>0$ on the left side and $g_{D} \leq 0$ on the right side. As Condition 4 shows, these inequalities are compatible when the coefficient $\Omega=(Y / K) /(R / W)$ is sufficiently high. (At least, $\Omega>1$, which is verified by (18).) When we fix $R / W$ in $\Omega$, high $\Omega$ means high capital/output ratio $Y / K$. Therefore, for sustainable growth, it is necessary to maintain a high productivity of capital, in spite of the accumulation of capital stock. This implies that sustainable growth needs a growth engine, as assumed in an endogenous growth theory. When we express $\Omega$ as

$$
\Omega=\frac{Y / R}{K / W},
$$

high $\Omega$ means that the output per weight is more dematerialized than the capital stock per weight $(\Omega \gg 1 \Rightarrow Y / R \gg K / W)$. Since part of output is invested and added to the capital stock, the capital is also being dematerialized. Therefore, in order to maintain high $\Omega$, the output should be further dematerialized as time goes on. This suggests that a growth engine sector should foster dematerialization, reducing its load to the environment.

Concluding the analysis of feasibility of sustainable growth, we would like to stress that these 
results are obtained without the detailed structure of the economy such as CIES utility function and Cobb-Douglas production function. Also, these results are not straightforward or trivial. In this point, this study is able to provide more general perspective of sustainable growth than previous prominent studies such as Stokey (1998) and Aghion and Howitt (1998), although detailed structure should be specified for further characterization of sustainable growth.

\section{Optimal sustainable growth}

In this section, we investigate an optimal sustainable growth path using an endogenous growth model with MBP. This study assumes that the total labor in the economy remains constant over time and is thus standardized to one. Economic growth affects consumer utility via consumption levels, and pollution affects consumers directly via disutility from the pollution stock. We assume that the instantaneous utility function is additively separable with respect to both consumption and pollution stock, and that elasticity of marginal utility is constant. In order to obtain an optimal steady state growth path, constancy of these elasticities over time is necessary (See Barro and Salai-Martin, 1995). These assumptions are quite usual in the literature of economic growth and the environment . There are identical households whose instantaneous utility function is given as

$$
U(C, D)=\frac{C^{1-\sigma}-1}{1-\sigma}-\gamma \frac{D^{1+\omega}}{1+\omega}, \quad \gamma, \sigma, \omega>0 \text { (constant) }
$$

This functional form follows Stokey (1998) and Aghion and Howitt (1998). The time discount rate is denoted by $\rho$, which is positive and constant. ${ }^{11}$

\footnotetext{
${ }^{11}$ The proofs for the propositions in this section, since long and routine, are thus omitted. These are available upon request.
} 


\subsection{Optimal sustainable growth without recycling}

In this subsection, we assume that there are no recycling activities, i.e. $v=1$. We take account of these recycling activities in the next subsection. Let

$$
\begin{aligned}
& Y=A K^{\alpha}\left(B L_{f}\right)^{\beta} R^{1-\alpha-\beta}, \quad A, \alpha, \beta>0 \text { (constant) }, \alpha+\beta<1, \\
& \dot{B}=\eta_{B} L_{B} B, \quad \eta_{B}>0 \text { (constant) }
\end{aligned}
$$

be the production functions of the final goods sector and the intermediate goods sector, respectively. ${ }^{12}$ Here, $A$ is the level of technology or quality index, $\eta_{B}$ is the fixed productivity parameter, and $B$ is the index of labor augmenting technology. The sector which produces $B$ can be the growth-engine of the economy. ${ }^{13} L_{f}$ and $L_{B}$ are labor inputs into the final goods sector and the growth-engine sector, respectively. We also assume, for simplicity, that the natural resource flow is extracted without cost.

\footnotetext{
${ }^{12}$ In his analysis of a general equilibrium model with the mass balance principle, Pethig (2005) shows that a production function should have the property in which the marginal productivity of materials is less than unity and points out that a Cobb-Douglas function does not satisfy such a requirement. He measures the product and the materials in terms of weight. The result follows from the fact that the weight of the product must be less than the weight of the (tangible) matrials. Our production function (25), although a Cobb-Douglas function, is not contradicted by Pethig's result, because we do not use weight as the unit of the product in (25).

${ }^{13}$ As well known, there are several economic interpretations of the growth-engine sector in the literature of endogenous growth theories. Correspondingly, the state variable $B$ may be human capital, knowledge, index of technology, variety of products, or so on. The important thing is that $B$ is intangible, and thus harmless to the environment. The necessity for such a sector is discussed at the end of the previous section.
} 
Then, the optimal growth problem is written:

$$
\begin{aligned}
&(\mathrm{P}) \max \int_{0}^{\infty}\left[\frac{C^{1-\sigma}-1}{1-\sigma}-\gamma \frac{D^{1+\omega}}{1+\omega}\right] e^{-\rho t} d t \\
& \text { subject to } Y=A K^{\alpha}\left(B L_{f}\right)^{\beta} R^{1-\alpha-\beta}, \\
& \dot{K}=Y-v(C+\delta K), \\
& \dot{B}=\eta_{B} L_{B} B, \\
& \dot{W}=-v\left(\frac{R}{Y} C+\delta W\right)+R, \\
& \dot{D}=v\left(\frac{R}{Y} C+\delta W\right)-\theta D, \\
& D \in\left(0, D_{\max }\right), L_{f}+L_{B}=1, v=1, \\
& K_{0}, B_{0}>0, \text { and } D_{0} \in\left(0, D_{\max }\right) \text { are given, }
\end{aligned}
$$

where control variables are $C, R, L_{f}$, and $L_{B}$.

Since our concern is the long-term consequences of economic growth, we focus on an optimal sustainable growth path. The following proposition shows the necessary conditions. It is derived from Pontryagin's maximum principle. Let $x_{0}^{*}$ be the initial value of the function of time $x(t)$, with which an optimal sustainable growth path begins.

Proposition 4. Consider the problem (P). (a) Necessary conditions for the existence of an optimal sustainable growth path are

$$
\begin{gathered}
\sigma \geq 1, \\
\eta_{B}>\rho, \\
\theta>-\frac{1-\sigma}{1+\omega} g_{K} .
\end{gathered}
$$


(b) The growth rates are characterized by

$$
\begin{gathered}
g_{K}=g_{Y}=g_{C}=\frac{\beta(1+\omega)\left(\eta_{B}-\rho\right)}{(1-\alpha)(1+\omega)-(1-\sigma)[(1-\alpha-\beta)+\beta(1+\omega)]}, \\
g_{B}=\frac{(1-\alpha)(\omega+\sigma)+\beta(1-\sigma)}{\beta(1+\omega)} g_{K}>g_{K}, \\
g_{R}=g_{D}=g_{W}=\frac{1-\sigma}{1+\omega} g_{K} \leq 0 .
\end{gathered}
$$

(c) If $\sigma>1$, the initial values of all variables are obtained as functions of $D_{0} \in\left(D_{\max }, 0\right)$. On the other hand, if $\sigma=1$, the triplet of the initial values $\left(B_{0}^{*}, Y_{0}^{*}, C_{0}^{*}\right)$ is obtained as a function of $K_{0}$, whereas $D_{0}^{*}$ is determined independently and the pair of $\left(W_{0}^{*}, R_{0}^{*}\right)$ is determined by $D_{0}^{*}$. Therefore, another necessary condition $D_{0}^{*}<D_{\max }$ is required when $\sigma=1$.

The necessary conditions show that:

(1) The intertemporal elasticity of substitution of consumption $\sigma^{-1}$ should be less than or equal to 1. Empirical evidence strongly supports the elasticity to be less than 1 (see Hall, 1988; Ogaki and Reinhart, 1998), although Aghion and Howitt (1998, p.162) caution that this condition results in odd behavior in some macroeconomic models.

(2) The growth-engine sector should be productive in the sense that the productivity parameter $\eta_{B}$ is higher than the discount rate $\rho$.

(3) The assimilation ability of nature $\theta$ should be sufficiently high to allow for the optimal rate of economic growth.

These necessary conditions are quite similar to those obtained by Stokey (1998) and Aghion and Howitt (1998). One technical reason for the similarity is that we commonly use an additively separable utility function. It is verified that on an optimal sustainable growth path, the instantaneous utilities (after an appropriate positive affine transformation) grow exponentially at a constant rate 
$\dot{U} / U=g_{U} \cdot{ }^{14}$ With the functional form of utility (24), this holds only if

$$
g_{U}=(1-\sigma) g_{C}=(1+\omega) g_{D}
$$

Therefore, sustainable growth $\left(g_{C}>0\right.$ and $\left.g_{D} \leq 0\right)$ are compatible only if $\sigma \geq 1$. Another technical reason is that natural resource flow $R$ works almost identically to Stokey's $z$, the intensity of pollution, in the final goods production. ${ }^{15}$

We should note, however, that unlike their models, the model $(\mathrm{P})$ takes into account the materials balance as well as the negative environmental effects of consumption. As a result, Proposition 4 illustrates how consumption changes on an optimal sustainable growth path: While the amount of consumption $C$ exponentially grows, the weight or the entity which harms the environment, $C R / Y$, decreases due to the reduction of the unit weight $R / Y$ with a more rapid speed than $C$. This follows from

$$
\left|g_{R / Y}\right|=\left|-\alpha g_{K}-\beta g_{B}+(\alpha+\beta) g_{R}\right|=\left|-\frac{\sigma+\omega}{1+\omega} g_{K}\right| \geq g_{C}
$$

using (27), (35), (36), and (37). The last inequality holds by $\sigma \geq 1$. Therefore, in a society which realizes sustainable growth as an optimal path, over time a higher level of well-being is brought by immaterial contributions.

\footnotetext{
${ }^{14}$ By the construction of Problem (P), the utility function (24) is unique up to a positive affine transformation. Also, notice that if $\sigma>1$, the utility takes a negative value with the upper limit, $\sup U=0$. Therefore, $g_{U}<0$ means that $U$ is increasing over time.

${ }^{15}$ The production function employed by Stokey (1998) has the form of $\tilde{y}=z y$, where $\tilde{y}$ and $y$ are the actural and potential outputs, respectively, and $z \in[0,1]$ is the intensity of pollution. Aghion and Howitt (1998) also adopted this formulation. The similarity is verified when we replace $R$ in $(25)$ with $z^{1 /(1-\alpha-\beta)}$. However, the processses with which $R$ and $z$ affect the environment are different: The environmental load is directly influenced by the choice of $z$, whereas the negative effect of $R$ is taken after it is transformed into final goods and they are consumed or disposed.
} 


\subsection{Optimal sustainable growth with recycling}

In this subsection, we incorporate recycling into the model. Assume that the recycling sector uses both waste and labor to produce an investment good. Let $q(v)$ be the labor input coefficient. That is, with $q(v)$ unit of labor, one unit of waste is made into $(1-v)$ unit of investment good. The effective labor $q(v)(C+\delta K)$ is required to attain recycling rate $(1-v)$. The demand for effective labor exponentially grows on a sustainable growth path. In other words, for an economy with a constant population, recycling can remain active on a sustainable growth path only if in the recycling sector labor augmenting technical progress lasts forever.

In order to quantify the technical progress, let $Q$ stand for levels of the recycling technology, which defines the demand for raw labor $L_{v}$ as

$$
L_{v}=q(v) \frac{(C+\delta K)}{Q}
$$

Since the labor supply is bounded, $Q$ needs to grow at least as fast as $C$ and $K$ on a steady state growth path:

$$
g_{Q} \geq g_{K}
$$

We assume that the recycling technology $Q$ evolves by

$$
\dot{Q}=\eta_{Q} L_{Q} Q, \quad \eta_{Q}>0 \text { (constant) }
$$

where $\eta_{Q}$ is the fixed recycling technology parameter and $L_{Q}$ is labor input into the R\&D activity for recycling. Also we assume that function $q:(0,1] \rightarrow \mathbb{R}_{+}$has the following properties:

$$
q^{\prime}(v)<0 \text { all } v \in(0,1), \quad q(1)=0, \text { and } \lim _{v \rightarrow 0} q(v)=\infty
$$


Then, problem $(\mathrm{P})$ is modified as follows:

$$
\begin{aligned}
& \left(\mathrm{P}^{\prime}\right) \quad \max \int_{0}^{\infty}\left[\frac{C^{1-\sigma}-1}{1-\sigma}-\gamma \frac{D^{1+\omega}}{1+\omega}\right] e^{-\rho t} d t \\
& \text { subject to }(27),(28),(29),(30),(31), \\
& \dot{Q}=\eta_{Q} Q\left[1-L_{B}-L_{f}-q(v) \frac{C+\delta K}{Q}\right], \\
& D \in\left(0, D_{\max }\right), \quad L_{f}+L_{B} \leq 1, \quad v \in(0,1] \\
& K_{0}, B_{0}, Q_{0}>0, \text { and } D_{0} \in\left(0, D_{\max }\right) \text { are given, }
\end{aligned}
$$

where control variables are $C, R, L_{f}, L_{B}$, and $v$.

For this problem, we have:

Proposition 5. For the problem $\left(P^{\prime}\right)$, if an interior optimal sustainable growth path exists, the following inequalities hold:

$$
\begin{gathered}
\sigma \geq 1, \\
1>\left(\eta_{B}^{-1}+\eta_{Q}^{-1}\right) \rho, \\
\theta>-\frac{1-\sigma}{1+\omega} g_{K} .
\end{gathered}
$$

Compared with Proposition 4, the different condition is (43). ${ }^{16}$ That is, with recycling activity, not only $\eta_{B}$, but also $\eta_{Q}$ needs to be higher than $\rho$. In fact, $\eta_{B}$ and $\eta_{Q}$ must jointly satisfy (43) since labor for the $R \& D$ is divided into production and environment management sectors. We need to note that technological progress is required in both production and recycling sectors to acknowledge

\footnotetext{
${ }^{16}$ This condition has not appeared in the literature on growth and recycling (Di Vita, 2001 and Pittel et al. 2005), since these studies abstract from the input to treat and recycle waste.
} 
the benefit of recycling activities so that we are able to use less resources to produce goods. As a result, we have the severer productivity condition than Proposition 4. Note, however, that even though the economy fails to satisfy (43), it may be still possible to have an optimal sustainable growth path without recycling. This indicates that recycling activity is not necessarily essential to realize an optimal sustainable growth.

\section{Concluding remarks}

Economists have largely focused on the analysis of pollution from the production side in the literature of economic growth and the environment (see Brock and Taylor, 2006, for review). This paper studies the feasibility and optimality of sustainable growth considering pollution from both the production and the consumption sides. We adopted a materials balance approach to the endogenous growth theory to comprehensively capture the system of the economy and the environment.

We find that sustainable growth is impossible, with and without recycling activities, if all waste affects the environment. The insight gained from this impossibility result is that we should distinguish between harmful and harmless waste. The materials balance approach is used to highlight the distinction between tangible and intangible goods (or harmful and harmless waste to the environment). Sustainable growth is shown to be feasible only if the growth rate of the natural resource flow, which is the primary form of tangible goods, is non-positive.

This result gives us a picture of sustainable growth. In an economy that enjoys sustainable growth, the dependence on materials extracted from nature should be reduced and people should gain their well-being more and more from dematerialization. An interesting agenda would be to investigate the long term trend of the weights of natural resources coming in and going out of the economy to evaluate the sustainability of said economy. Already, Ayres and van den Bergh (2005) 
have done this for resource inputs in the US and show a decreasing trend between 1900 and 1998 .

In order that sustainable growth is feasible and optimal, the assimilation ability of nature should be sufficiently high to allow for an optimal rate of economic growth. Note that material flows which return back to the environment normally have long term environmental consequences. Usual examples are chemically stable substances. These include greenhouse gases such as carbon dioxide, nuclear or organic waste, or certain toxic substances like PCB. These are accumulated as pollution stock in the environment and difficult to decompose by natural processes so that the associated assimilation coefficient is low. For an economy which emits a mass of chemically stable substances, sustainable growth may not be optimal.

Finally, the results obtained in this paper need to be interpreted in the long run. For example, recycling will be inactive on an optimal sustainable development path if the technological progress of recycling is not rapid, or if the productivity of the $\mathrm{R} \& \mathrm{D}$ for recycling is not high. This, however, does not imply that recycling is unnecessary. Recycling may be essential in the transitional period to an optimal sustainable growth path. The transition dynamics to and the stability of an optimal sustainable growth path are left to future research. 


\section{Appendix: Proof of Lemma 1}

For the proof, the following sublemma is useful:

Sublemma 1. Suppose that $A_{1} A_{2} A_{3} \neq 0$ and the following equation holds:

$$
A_{1} e^{a_{1} t}+A_{2} e^{a_{2} t}+A_{3} e^{a_{3} t}=g \text { (constant) all } t \geq 0 .
$$

Then, (i) $a_{1}=a_{2}=a_{3}=a$ and $a\left(A_{1}+A_{2}+A_{3}\right)=0$, or (ii) $a_{j}=a_{k} \neq a_{i}=0, A_{j}=-A_{k}$, and $A_{i}=g$, where $i, j, k \in\{1,2,3\}$.

Proof. Differentiate (45) with respect to time:

$$
a_{1} A_{1} e^{a_{1} t}+a_{2} A_{2} e^{a_{2} t}+a_{3} A_{3} e^{a_{3} t}=0 .
$$

We consider two cases: (a) any of $a_{i}$ is equal to zero $\left(a_{1} a_{2} a_{3}=0\right)$, and (b) none of $a_{i}$ is equal to zero $\left(a_{1} a_{2} a_{3} \neq 0\right), a_{i} \neq 0$. Case (a) Without loss of generality, we can assume $a_{1}=0$. If also $a_{2}=0$, $a_{3}=0$ by (46) and we have the case (i). If $a_{2} \neq 0,(46)$ becomes

$$
a_{2} A_{2} e^{a_{2} t}\left(1+\frac{a_{3} A_{3}}{a_{2} A_{2}} e^{\left(a_{3}-a_{2}\right) t}\right)=0 .
$$

Since $a_{2} A_{2} e^{a_{2} t} \neq 0$, it is the case (ii). Case (b) Since $a_{1} \neq 0,(46)$ becomes

$$
\frac{a_{2} A_{2}}{a_{1} A_{1}} e^{\left(a_{2}-a_{1}\right) t}+\frac{a_{3} A_{3}}{a_{1} A_{1}} e^{\left(a_{3}-a_{1}\right) t}=-1 .
$$

Differentiate this with respect to time and we have

$$
\left(a_{2}-a_{1}\right) \frac{a_{2} A_{2}}{a_{1} A_{1}} e^{\left(a_{2}-a_{1}\right) t}+\left(a_{3}-a_{1}\right) \frac{a_{3} A_{3}}{a_{1} A_{1}} e^{\left(a_{3}-a_{1}\right) t}=0 .
$$


If $a_{2}-a_{1}=0$, also $a_{3}-a_{1}=0$. Thus, from (46), we have the case (i). If $a_{2}-a_{1} \neq 0,(47)$ implies

$$
1+\left(\frac{a_{3}-a_{1}}{a_{2}-a_{1}}\right) \frac{a_{3} A_{3}}{a_{2} A_{2}} e^{\left(a_{3}-a_{2}\right) t}=0 .
$$

Therefore, we have $a_{2}=a_{3} \neq a_{1}$ and $A_{2}=A_{3}$. But, substitution of these results into (46) yields $a_{1}=0$, a contradiction. That is, $a_{1} a_{2} a_{3} \neq 0$ implies the case (i).

\section{Proof of Lemma 1.}

Consider a steady state growth path. (1) is rewritten as

$$
g_{K}=\frac{Y_{0}}{K_{0}} e^{a_{1} t}-v_{0} \frac{C_{0}}{K_{0}} e^{a_{2} t}-\delta v_{0} e^{a_{3} t}
$$

where $a_{1}=g_{Y}-g_{K}, a_{2}=g_{v}+g_{C}-g_{K}$, and $a_{3}=g_{v} \leq 0$. First, consider the case (ii) of Sublemma

1. The correspondence for $A_{i}$ and $g_{K}$ in the sublemma is

$$
A_{1}=\frac{Y_{0}}{K_{0}}>0, \quad A_{2}=-v_{0} \frac{C_{0}}{K_{0}}<0, \quad A_{3}=-\delta v_{0}<0, \quad g=g_{K} .
$$

Since $g_{K}<0$ is ruled out on a steady state growth path, the possible case is $A_{1}=g_{K}$. However, this implies $C_{0} / K_{0}=-\delta<0$, a contradiction. Next, consider the case (i). Suppose $a_{1}=a_{2}=a_{3} \neq 0$. This implies $g_{K}=0$. Then, we have $g_{Y}=a_{1}=a_{3}=g_{v}<0$, which contradicts the definition of steady state growth. Therefore, $a_{1}=a_{2}=a_{3}=0$, and we thus have $g_{Y}=g_{K}=g_{C}$ and $g_{v}=0$. Finally, consider the case of $g_{v}<0$. For the case (i), $g_{v} \neq 0$ implies $A_{1}+A_{2}+A_{3}=g_{K}=0$, which in turn implies $g_{Y}=a_{1}=a_{3}=g_{v}<0$. For the case (ii), as seen just above, it must be the case that $a_{1}=a_{3} \neq a_{2}=0$ and $g_{K}=A_{2}<0$. 


\section{References}

[1] Aghion, P, Howitt, P., 1998. Endogenous Growth Theory, MIT Press, Cambridge, MA.

[2] Andreoni, J., Levinson, A., 2001. The simple analytics of the environmental Kuznets curve. Journal of Public Economics, 80, 269-286.

[3] Asheim, G.B., Buchholz, W., Tungodden, B., 2001. Justifying Sustainability. Journal of Environmental Economics and Management, 41, 252-268.

[4] Ayres, R.U., 1999. Materials, economics and the environment, in: van den Bergh, J.C.J.M. (Ed.), Handbook of Environmental and Resource Economics, Edward Elgar, Chelten-ham, UK, pp. $867-894$.

[5] Ayres, R.U., and Kneese, A.V., 1969. Production, consumption, and externalities. American Economic Review 59, 282-297.

[6] Ayres, R.U. and van den Bergh, J.C.J.M., 2005. A theory of economic growth with material/energy resources and dematerialization: interaction of three growth mechanisms. Ecological Economics 55, 96-118.

[7] Barro, R.J. Sala-i-Martin, X., 1995. Economic Growth, MIT Press, Cambridge, MA.

[8] Bovenberg, A.L., Smulders, S., 1995. Environmental quality and pollution-augmenting technological change in a two-sector endogenous growth model. Journal of Public Economics 57, 369-391.

[9] Brock, W., Taylor, M.S., 2006. Economic growth and the environment: a review of theory and empirics. in: Durlauf, S., Aghion., P. (Eds.), The Handbook of Economic Growth, Amsterdam, North Holland. 
[10] Di Vita, G., 2001. Technological Change, Growth and Waste Recycling, Energy Economics 23, $549-567$.

[11] Eichner, T., Pethig, R. 2001. Product design and efficient management of recycling and waste. treatment. Journal of Environmental Economics and Management 41, 109-34.

[12] Hall, R.E., 1988. Intertemporal substitution in consumption. Journal of Political Economy 96, $339-357$.

[13] Hartman, R. Kwon., O.-S., 2005. Sustainable growth and the environmental Kuznets curve, Journal of Economic Dynamics and Control 29, 1701-1736.

[14] Heal, G., 1998. Valuing the Future: Economic Theory and Sustainability. Columbia University Press, New York.

[15] Jones, L.E., Manuelli, R.E., 2001. Endogenous policy choice: the case of pollution and growth. Review of Economic Dynamics 4, 369-405.

[16] Mäler, K.-G. 1974. Environmental Economics: A Theoretical Inquiry. Johns Hopkins University Press, Baltimore, MD.

[17] Ogaki, M., Reinhart, C.M., 1998. Measuring intertemporal substitution: the role of durable goods. Journal of Political Economy 106, 1078-1098.

[18] Pethig, R., 2005. Non-linear production, abatement, pollution and materials balance reconsidered. Journal of Environmental Economics and Management, forthcoming.

[19] Pezzey, J., 1992. Sustainability: an interdisciplinary guide. Environmental Values 1, 321-362.

[20] Pezzey, J., 1997. Sustainability constraints versus "Optimality" versus intertemporal concern, and axioms versus data. Land Economics, 73, 448-466. 
[21] Pittel, K., Jean-Pierre, A. and Kuhn, T., 2005. Endogenous growth and recycling: A material balance approach, Economics Working Papers Series 05/37, ETH Zurich

[22] Smith, V.L., 1972. Dynamics of waste accumulation: disposal versus recycling. Quarterly Journal of Economics 86, 600-616.

[23] Smulders, S., 2000. Economic growth and environmental quality. in: Folmer, H., Gabel, L. (Eds.), Principles of Environmental Economics, Cheltenham: Edward Elgar, chapter 20, 602664.

[24] Stokey, N.L., 1998. Are there limits to growth? International Economic Review 39, 1-31.

[25] United Nations, 1993. Agenda 21: programme of action for sustainable development. United Nations Dept. of Public Information, New York.

[26] Van den Bergh, J.C.J.M. and Nijkamp, P., 1994. Dynamic macro modelling and materials balance: economic-environmental integration for sustainable development, Economic Modelling 11, 283-307.

[27] World Commission on Environment and Development, 1987. Our Common Future. Oxford University Press, New York.

[28] Xepapadeas, A., 2003. Economic growth and environment, in: Mäler, K.-G. and Vincent, J. (eds.), Handbook of Environmental Economics, Vol. 3. North-Holland, Amsterdam, forthcoming. 


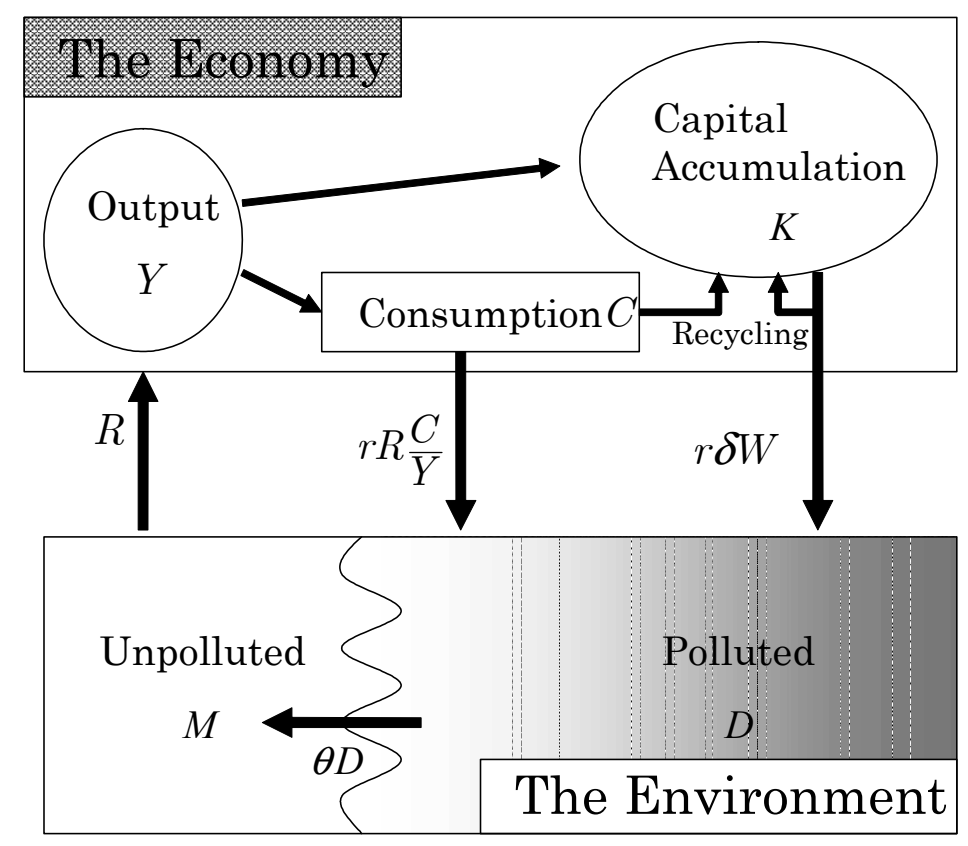

Figure 1: Materials Balance of the Economy and the Environment.

$<$ Figure $>$ 


\section{Appendix For Referees:}

\section{A Proof of Lemma 1, Proposition 2 and 3}

Please see the main text and the Appendix.

\section{B Proof of Proposition 4}

The associated Hamiltonian is defined by

$$
\begin{aligned}
H= & {\left[\frac{C^{1-\sigma}-1}{1-\sigma}-\gamma \frac{D^{1+\omega}}{1+\omega}\right] } \\
& +\lambda[Y-(C+\delta K)]+\mu \eta_{B}\left(1-L_{f}\right) B \\
& +\xi\left[-\left(\frac{R}{Y} C+\delta W\right)+R\right]+(-\zeta)\left[\left(\frac{R}{Y} C+\delta W\right)-\theta D\right],
\end{aligned}
$$

where $\lambda, \mu, \xi$, and $-\zeta$ are the costate variables corresponding to state variables, $K, B, W$, and $D$, respectively. Pontryagin's maximum principle is written as follows:

1. Maximum conditions

$$
\begin{gathered}
\frac{\partial H}{\partial C}=C^{-\sigma}-\lambda-(\xi+\zeta) \frac{R}{Y}=0, \\
\frac{\partial H}{\partial L_{f}}=\beta \lambda \frac{Y}{L_{f}}-\mu \eta_{B} B+\beta(\xi+\zeta) \frac{R C}{Y L_{f}}=0, \\
\frac{\partial H}{\partial R}=\lambda(1-\alpha-\beta) \frac{Y}{R}-(\alpha+\beta)(\xi+\zeta) \frac{C}{Y}+\xi=0 .
\end{gathered}
$$


2. Adjoint equations

$$
\begin{aligned}
& \dot{\lambda}=\rho \lambda-\left\{\lambda\left(\alpha \frac{Y}{K}-\delta\right)+(\xi+\zeta) \alpha \frac{R C}{Y K}\right\}, \\
& \dot{\mu}=\rho \mu-\left\{\beta \lambda \frac{Y}{B}+\mu \eta_{B}\left(1-L_{f}\right)+\beta(\xi+\zeta) \frac{R C}{Y B}\right\}, \\
& \dot{\xi}=\rho \xi+(\xi+\zeta) \delta, \\
& \dot{\zeta}=(\rho+\theta) \zeta-\gamma D^{\omega} .
\end{aligned}
$$

Suppose that $\mu=0$. Then, from (50)

$$
\lambda Y / C=-(\xi+\zeta) R / Y
$$

From this and (49), $g_{\lambda}=-\sigma g_{C}<0$. (Recall that $g_{Y}=g_{C}>0$ on a sustainable growth path by the definition and Lemma 1.) On the other hand, substitution of (56) into (52) yields $g_{\lambda}=\rho+\delta>0$. This is a contradiction and thus $\mu \neq 0$. Rewrite (50) as

$$
\beta \lambda \frac{Y}{B}-\mu \eta_{B} L_{f}+\beta(\xi+\zeta) \frac{R C}{Y B}=0
$$

Combine this with (53) to obtain

$$
g_{\mu}=\rho-\eta_{B} \cdot(\text { constant })
$$

Substitute (57) into (53) to obtain

$$
\frac{\beta \lambda Y}{\mu B}-\eta_{B} L_{f}+\beta(\xi+\zeta) \frac{R C}{Y \mu B}=0
$$


Substitute (58) into (52) to obtain

$$
\dot{\lambda}=(\rho+\delta) \lambda-\frac{\alpha \eta_{B} L_{f}}{\beta} \frac{\mu B}{K}
$$

On the other hand, substitute (58) into (49) to obtain

$$
\left(1-\frac{Y}{C}\right) \lambda=C^{-\sigma}-\frac{\eta_{B} L_{f}}{\beta} \frac{\mu B}{C}
$$

Differentiate (60) with respect to time. Recall $g_{K}=g_{C}=g_{Y}$ along a sustainable growth path. We have

$$
\left(1-\frac{Y}{C}\right) \dot{\lambda}=-\sigma C^{-\sigma} g_{K}-\frac{\eta_{B} L_{f}}{\beta} \frac{\mu B}{C}\left(g_{\mu}+g_{B}-g_{K}\right) .
$$

Substitute (60) into (59) to obtain

$$
\left(1-\frac{Y}{C}\right) \dot{\lambda}=(\rho+\delta)\left(C^{-\sigma}-\frac{\eta_{B} L_{f}}{\beta} \frac{\mu B}{C}\right)-\left(1-\frac{Y}{C}\right) \frac{\alpha \eta_{B} L_{f}}{\beta} \frac{\mu B}{K} .
$$

Combine (61) with (62) and we have

$$
\left(\rho+\delta+\sigma g_{K}\right) \frac{C^{1-\sigma}}{\mu B}-\frac{\eta_{B} L_{f}}{\beta}\left(\rho-g_{\mu}-g_{B}+\left(g_{K}+\delta\right)(1-\alpha)\right)=0 .
$$

This implies that $C^{1-\sigma} /(\mu B)$ is constant, i.e.

$$
g_{B}=(1-\sigma) g_{K}-g_{\mu} \cdot(\text { constant })
$$

Since $(60)$ is rearranged as

$$
\left(1-\frac{Y}{C}\right) \frac{\lambda C}{\mu B}=\frac{C^{1-\sigma}}{\mu B}-\frac{\eta_{B} L_{f}}{\beta}
$$


(64) implies that $\lambda C /(\mu B)$ is constant, so that ${ }^{17}$

$$
g_{\lambda}=g_{\mu}+g_{B}-g_{K}=-\sigma g_{K} \cdot(\text { constant })
$$

Since $\lambda Y /(\mu B)$ and $C / Y$ are constant, (58) yields

$$
g_{\xi+\zeta}=g_{\mu}+g_{B}-g_{R}=g_{K}+g_{\lambda}-g_{R}=(1-\sigma) g_{K}-g_{R} \text {. (constant) }
$$

(66) implies that $\lambda Y / R$ has constant growth rate $g_{\xi+\zeta}$. Then, (51) implies $g_{\xi+\zeta}=g_{\xi}$ if $\xi \neq 0$. By (54) and (55), we can verify $\xi \neq 0$ and have

$$
g_{\xi+\zeta}=g_{\xi}=g_{\zeta}=\omega g_{D} .(\text { constant })
$$

It follows from (66) and (67) that

$$
g_{D}=\frac{1-\sigma}{1+\omega} g_{K}
$$

Note that $g_{D} \leq 0$ on a sustainable path by Proposition 3. Therefore, (68) implies $\sigma \geq 1$, the necessary condition (32).

As for the condition for assimilation ability, (31) is rewritten as:

$$
\frac{v}{D}\left(\frac{R C}{Y}+\delta W\right)=g_{D}+\theta>0
$$

Thus, we obtain the environmental constraint (34):

$$
\theta>-g_{D}=\frac{\sigma-1}{1+\omega} g_{K} .
$$

\footnotetext{
${ }^{17}$ Note $1-Y / C=-\left(g_{K}+\delta\right) K / C \neq 0$.
} 
Using (27) and (68), we have:

$$
g_{B}=\frac{(1+\omega)(1-\alpha)+(\sigma-1)(1-\alpha-\beta)}{\beta(1+\omega)} g_{K}
$$

(Note $g_{Y}=g_{K}$ and $g_{R}=g_{D}$ by Proposition 3.) Combine (69), (57), and (64) to obtain

$$
g_{K}=\frac{\beta(1+\omega)\left(\eta_{B}-\rho\right)}{(1-\alpha)(1+\omega)+(\sigma-1)[(1-\alpha-\beta)+\beta(1+\omega)]}
$$

Therefore, on the premise of $\sigma \geq 1, g_{K}>0$ if and only if $g_{\mu}=\rho-\eta_{B}<0$. This is the necessary condition (33).

We have so far characterized all growth rates as well as the necessary conditions (32) - (34) in Proposition 4. Now we turn to the characterization of the initial values for the variables. Let us start with the optimal labor input into the final sector $L_{f}$. Since $L_{f}=1-L_{B}=1-g_{B} / \eta_{B}<1$ by (29), we have only to check its positivity. Using (57) and (64), we have

$$
L_{f}^{*}=1-\frac{(1-\sigma) g_{K}-\left(\rho-\eta_{B}\right)}{\eta_{B}}=\frac{(\sigma-1) g_{K}+\rho}{\eta_{B}}>0
$$

since $\sigma \geq 1$. For expositional convenience, we introduce additional notation:

$$
\pi=\xi+\zeta
$$

and

$$
\kappa=\frac{\delta}{g_{\xi}-\rho} .
$$


Note $\kappa<0$ by (67) and $\xi=\kappa \pi$ by (54). Using these, (49) - (52) are rewritten as follows:

$$
\begin{aligned}
& 1+\frac{\pi R C}{\lambda Y^{2}}=\frac{C^{1-\sigma}}{\lambda Y}+\frac{K}{Y}\left(g_{K}+\delta\right), \\
& 1+\frac{\pi R C}{\lambda Y^{2}}=\frac{\eta_{B} L_{f}}{\beta} \frac{\mu B}{\lambda Y}, \\
& 1+\frac{\pi R C}{\lambda Y^{2}}=(\alpha+\beta)^{-1}\left(1+\kappa \frac{\pi R}{\lambda Y}\right), \\
& 1+\frac{\pi R C}{\lambda Y^{2}}=\frac{\rho+\delta-g_{\lambda}}{\alpha} \frac{K}{Y} .
\end{aligned}
$$

Here, (72) is derived by

$$
\frac{C}{Y}=\frac{K}{Y}\left(\frac{Y-\delta K-\dot{K}}{K}\right)=1-\frac{K}{Y}\left(\delta+g_{K}\right)
$$

which follows from (28).

By (74) and (75),

$$
\kappa \frac{\pi R C}{\lambda Y^{2}}=\left[\frac{(\alpha+\beta)\left(\rho+\delta-g_{\lambda}\right)}{\alpha} \frac{K}{Y}-1\right] \frac{C}{Y}
$$

Substitute this and (76) into (72) to yield

$$
\frac{C^{1-\sigma}}{\lambda Y}=-\frac{K}{Y}\left(g_{K}+\delta\right)+1+\frac{1}{\kappa}\left[1-\frac{K}{Y}\left(\delta+g_{K}\right)\right]\left[\frac{(\alpha+\beta)\left(\rho+\delta-g_{\lambda}\right)}{\alpha} \frac{K}{Y}-1\right]
$$

On the other hand, combine (72) and (75) to obtain

$$
\frac{C^{1-\sigma}}{\lambda Y}=\left[\frac{\rho+\delta-g_{\lambda}}{\alpha}-\left(\delta+g_{K}\right)\right] \frac{K}{Y}
$$


By (77) and (78), we have the quadratic equation for $(K / Y): f(/ Y)=0$, where

$$
f(x):=P x^{2}+Q x+\alpha(1-\kappa)
$$

and

$$
\begin{aligned}
& P=\left(\delta+g_{K}\right)(\alpha+\beta)\left(\rho+\delta-g_{\lambda}\right)>0 \\
& Q=\kappa\left(\rho+\delta-g_{\lambda}\right)-\alpha\left(\delta+g_{K}\right)-(\alpha+\beta)\left(\rho+\delta-g_{\lambda}\right)<0 .
\end{aligned}
$$

This equation determines a unique solution of $(K / Y)$. This is verified as follows. First, notice that $f(0)=\alpha(1-\kappa)>0$. Second, observe the following inequality:

$$
f\left(\frac{1}{\delta+g_{K}}\right)=\kappa\left(\frac{\rho+\delta-g_{\lambda}}{\delta+g_{K}}-\alpha\right)<0
$$

since $\kappa<0,-g_{\lambda}=\sigma g_{K} \geq g_{K}$, and $\alpha<1$. Finally, notice that $(K / Y)$ must be less than $1 /\left(\delta+g_{K}\right)$ because $g_{K}+\delta+C / K=Y / K$.

Let $(K / Y)^{*}$ be the capital/output ratio obtained from (79). Then, using (70) and (76), we can also determine the optimal consumption/output ratio:

$$
(C / Y)^{*}=1-(K / Y)^{*}\left(\delta+g_{K}\right)
$$

We will use notation $(\cdot / \cdot)^{*}$ for the ratio derived from $(K / Y)^{*}$ and $(C / Y)^{*}$. For example, $(C / K)^{*}=$ $(K / Y)^{*} /(C / Y)^{*}$ 
Now we are ready to characterize the initial values of the all variables. Fix $D_{0}^{*} \in\left(0, D_{\max }\right)$. Then the associated initial values $W_{0}^{*}$ and $R_{0}^{*}$ are determined as follows. First, by (16) and (17),

$$
R_{0}^{*}=\frac{g_{R}+\delta}{g_{K}+\delta}\left(\frac{Y}{K}\right)^{*} W_{0}^{*} .
$$

Second, substitute this into (15) and solve it with respect to $W_{0}$ :

$$
W_{0}^{*}=\frac{\left(g_{R}+\theta\right)\left(g_{K}+\delta\right)}{\left(g_{R}+\delta\right)(Y / K)^{*}+\left(g_{K}+\delta\right) g_{R}} D_{0}^{*}
$$

$\zeta_{0}^{*}$ is determined from (55) and (67). Then, $\zeta_{0}^{*}$ determines $\xi_{0}^{*}$ by $(54)$. That is,

$$
\zeta_{0}^{*}=\frac{\left(D_{0}^{*}\right)^{\omega}}{\rho+\theta-\omega g_{R}} \text { and } \xi_{0}^{*}=\frac{-\delta \zeta_{0}^{*}}{\rho+\delta-\omega g_{R}} .
$$

Define

$$
b_{1}=\frac{\rho+(1-\alpha) \delta+(\sigma-\alpha) g_{K}}{\alpha}\left(\frac{C}{K}\right)^{* \sigma-1} .
$$

Then, from (78),

$$
b_{1}=\left(K_{0}^{*}\right)^{-\sigma} / \lambda_{0}^{*} .
$$

Next, from (73) and (75), we have

$$
b_{2}=\frac{\beta\left(\rho+\delta+\sigma g_{K}\right)}{\alpha \eta_{B} L_{f}}=\frac{\mu_{0}^{*} B_{0}^{*}}{\lambda_{0}^{*} K_{0}^{*}} .
$$

Then, using (75), define

$$
b_{3}=\left[\frac{\rho+\delta+\sigma g_{K}}{\alpha}-\left(\frac{Y}{K}\right)^{*}\right] \frac{(Y / C)^{*}}{\pi_{0}^{*} R_{0}^{*}}=\frac{1}{\lambda_{0}^{*} K_{0}^{*}},
$$


where $\pi_{0}^{*}=\xi_{0}^{*}+\zeta_{0}^{*}$, which is determined by (83). Finally, recall (25) and we have

$$
b_{4}=\left(\frac{K}{Y}\right)^{*} \frac{\left(R_{0}^{*}\right)^{\alpha+\beta-1}}{A\left(L_{f}^{*}\right)^{\beta}}=\left(K_{0}^{*}\right)^{\alpha-1}\left(B_{0}^{*}\right)^{\beta} .
$$

Rewrite the system of equations (84) - (87): ${ }^{18}$

$$
\left[\begin{array}{cccc}
-\sigma & 0 & -1 & 0 \\
-1 & 1 & -1 & 1 \\
-1 & 0 & -1 & 0 \\
\alpha-1 & \beta & 0 & 0
\end{array}\right]\left(\begin{array}{c}
\ln K_{0}^{*} \\
\ln B_{0}^{*} \\
\ln \lambda_{0}^{*} \\
\ln \mu_{0}^{*}
\end{array}\right)=\left(\begin{array}{c}
\ln b_{1} \\
\ln b_{2} \\
\ln b_{3} \\
\ln b_{4}
\end{array}\right)
$$

The determinant of the matrix on the left side is $(1-\sigma) \beta$. Therefore, if $\sigma>1$, the state and the costate variables are uniquely determined, so that we can obtain the initial values of all variables as functions of $D_{0}^{*} \in\left(0, D_{\max }\right)$.

If $\sigma=1, b_{1}=b_{3}$, i.e.,

$$
\pi_{0}^{*} R_{0}^{*}=\left[\frac{\rho+\delta+g_{K}}{\alpha}\left(\frac{Y}{K}\right)^{*}-1\right]\left[\frac{\rho+(1-\alpha) \delta+(1-\alpha) g_{K}}{\alpha}\right]^{-1}
$$

Using (81), (82), and (83), the left side of this equation is rewritten as

$$
\pi_{0}^{*} R_{0}^{*}=\frac{\left(\rho-\omega g_{R}\right)\left(g_{R}+\delta\right)\left(g_{R}+\theta\right)\left(D_{0}^{*}\right)^{\omega+1}}{\left(\rho+\delta-\omega g_{R}\right)\left(\rho+\theta-\omega g_{R}\right)\left[\left(g_{R}+\delta\right)+\left(g_{K}+\delta\right)(K / Y)^{*}\right]},
$$

and thus (88) determines $D_{0}^{*}$, which in turn determines $R_{0}^{*}$ and $W_{0}^{*}$. Notice that $g_{R}=g_{D}=g_{W}=0$ for the case of $\sigma=1$. Therefore, we have to add the necessary condition that $D_{0}^{*} \leq D_{\max }$. The

\footnotetext{
${ }^{18}$ Note that $b_{i}, i=1,2,3,4$ are positive and their values are known from the results previously obtained.
} 
choice of $K_{0}^{*}$ is arbitrary, and $B_{0}^{*}, \lambda_{0}^{*}$, and $\mu_{0}^{*}$ are determined by $K_{0}^{*}$ :

$$
\left[\begin{array}{ccc}
1 & -1 & 1 \\
0 & -1 & 0 \\
\beta & 0 & 0
\end{array}\right]\left(\begin{array}{c}
\ln B_{0}^{*} \\
\ln \lambda_{0}^{*} \\
\ln \mu_{0}^{*}
\end{array}\right)=\left(\begin{array}{c}
\ln b_{2}+\ln K_{0}^{*} \\
\ln b_{3}+\ln K_{0}^{*} \\
\ln b_{4}+(1-\alpha) \ln K_{0}^{*}
\end{array}\right)
$$

Notice that the determinant of the matrix on the left side is $\beta(\neq 0)$. We have thus proved the proposition.

\section{Proof of Proposition 5}

Modifying the Hamiltonian in the proof of Proposition 4, we define the relevant Hamiltonian by

$$
\begin{aligned}
H= & {\left[\frac{C^{1-\sigma}-1}{1-\sigma}-\gamma \frac{D^{1+\omega}}{1+\omega}\right]+\lambda[Y-v(C+\delta K)] } \\
& +\mu \eta_{B} L_{B} B+v \eta_{Q}\left[\left(1-L_{B}-L_{f}\right) Q-q(v)(C+\delta K)\right] \\
& -\xi\left[v\left(\frac{R}{Y} C+\delta W\right)-R\right]-\zeta\left[v\left(\frac{R}{Y} C+\delta W\right)-\theta D\right],
\end{aligned}
$$

where $\lambda, \mu, v, \xi$, and $\zeta$ are the costate variables corresponding to state variables, $K, B, Q, W$, and $D$, respectively.

If an interior optimal path exists, the following conditions must hold along the path: 
1. Maximum conditions

$$
\begin{aligned}
\frac{\partial H}{\partial C} & =C^{-\sigma}-\lambda v-v \eta_{Q} q(v)-(\xi+\zeta) v \frac{R}{Y}=0, \\
\frac{\partial H}{\partial L_{f}} & =\lambda \beta \frac{Y}{L_{f}}-v \eta_{Q} Q+\beta(\xi+\zeta) v \frac{R C}{Y L_{f}}=0, \\
\frac{\partial H}{\partial L_{B}} & =\mu \eta_{B} B-v \eta_{Q} Q=0, \\
\frac{\partial H}{\partial R} & =\lambda(1-\alpha-\beta) \frac{Y}{R}+\xi-(\xi+\zeta)(\alpha+\beta) v \frac{C}{Y}=0, \\
\frac{\partial H}{\partial v} & =-\lambda(C+\delta K)-v \eta_{Q} q^{\prime}(v)(C+\delta K)-(\xi+\zeta)\left(\frac{R}{Y} C+\delta W\right)=0 .
\end{aligned}
$$

2. Adjoint equations

$$
\begin{aligned}
& \dot{\lambda}=\rho \lambda-\left\{\lambda\left(\alpha \frac{Y}{K}-v \delta\right)-v \eta_{Q} q(v) \delta+(\xi+\zeta) \alpha \frac{v R C}{Y K}\right\}, \\
& \dot{\mu}=\rho \mu-\left\{\lambda \beta \frac{Y}{B}+\mu \eta_{B} L_{B}+(\xi+\zeta) \beta \frac{v R C}{Y B}\right\}, \\
& \dot{v}=v\left[\rho-\eta_{Q}\left(1-L_{f}-L_{B}\right)\right], \\
& \dot{\xi}=\rho \xi+(\xi+\zeta) v \delta=(\rho+v \delta) \xi+v \delta \zeta, \\
& \dot{\zeta}=(\rho+\theta) \zeta-\gamma D^{\omega} .
\end{aligned}
$$

Combine (90) and (93) to cancel out the terms with $(\xi+\zeta)$ and we have

$$
\lambda \beta \frac{C}{L_{f}}\left[\left(\frac{Y-v(C+\delta K)}{Y}\right)+\delta \frac{Y W}{C R}\right]-v \eta_{Q} Q\left[\left(\frac{C}{Y}+\delta \frac{W}{R}\right)+q^{\prime}(v) \frac{\beta v(C+\delta K)}{L_{f} Q} \frac{C}{Y}\right]=0
$$

Notice that $g_{K} \geq 0$ implies that the first bracket on the left side of (99) is positive. Therefore, if $v(t)=0, \lambda(t)=0$. Then, from (90), $\xi(t)+\zeta(t)=0$ follows. Substitute these into (89) to see a contradiction, $C=0$. Thus, $v \neq 0$ so that $g_{v}$ is well defined. On a sustainable growth path, 
$g_{R}=g_{D}=g_{W}(15)$ and $g_{Y}=g_{K}=g_{C}(18)$. Also, (38) holds with equality: $g_{Q}=g_{K}$ so that $L_{Q}$ is constant. Therefore, (99) implies

$$
g_{\lambda}=g_{v} \cdot(\text { constant })
$$

Substitute (100) into (90) to obtain

$$
\xi+\zeta=-\left(\lambda_{0} \beta \frac{Y_{0}}{L_{f}}-v_{0} \eta_{Q} Q_{0}\right)\left(\frac{Y_{0} L_{f}}{\beta v R_{0} C_{0}}\right) e^{\left(g_{\lambda}-g_{R}+g_{K}\right) t}
$$

Therefore, we have

$$
g_{\xi+\zeta}=g_{\lambda}-g_{R}+g_{K} \cdot(\text { constant })
$$

Moreover, substitution of (101) into (92) yields

$$
\xi=\left[\left(\xi_{0}+\zeta_{0}\right)(\alpha+\beta) v \frac{C}{Y}-\lambda_{0}(1-\alpha-\beta) \frac{Y_{0}}{R_{0}}\right] e^{\left(g_{\lambda}-g_{R}+g_{K}\right) t}
$$

Thus, we have

$$
g_{\xi}=g_{\zeta}=g_{\xi+\zeta} .(\text { constant })
$$

Applying these results to (89), we obtain

$$
\left(C_{0}\right)^{-\sigma} e^{-\sigma g_{K} t}=\left[v \lambda_{0}+v_{0} \eta_{Q} q(v)-\left(\xi_{0}+\zeta_{0}\right) v \frac{R_{0}}{Y_{0}}\right] e^{g_{\lambda} t}
$$

From this it follows that

$$
-\sigma g_{K}=g_{\lambda} \cdot(\text { constant })
$$

(98) is rewritten as

$$
g_{\zeta}=(\rho+\theta)-\gamma D^{\omega} / \zeta
$$


which is constant by (102), and thus

$$
g_{\zeta}=\omega g_{D} \leq 0
$$

Recall $g_{D}=g_{R}$, and combine (101), (102), (103), and (104). Then, we have

$$
g_{R}=\frac{1-\sigma}{1+\omega} g_{K}
$$

Therefore, $g_{K}>0$ and $g_{R} \leq 0$ are compatible only if $\sigma \geq 1$, which is the necessary condition (42). As for the condition for assimilation ability, rewrite (31) as:

$$
\frac{v}{D}\left(\frac{R C}{Y}+\delta W\right)=g_{D}+\theta>0
$$

and we have condition (44):

$$
\theta>-g_{D}=-\frac{1-\sigma}{1+\omega} g_{K}
$$

Finally, the productivity condition for the indices of technology (43) is derived as follows. By (100) and (103), $g_{v}=g_{\lambda}=-\sigma g_{K}<0$. From (96), we have

$$
1-L_{f}-L_{B}>\eta_{Q}^{-1} \rho
$$

On the other hand, (91) yields $g_{\mu}=g_{\lambda}+g_{K}-g_{B}<0 .{ }^{19}$ This inequality and (95) imply

$$
\rho<\frac{\lambda \beta Y}{\mu B}+\eta_{B} L_{B}+\frac{(\xi+\zeta) \beta v R C}{\mu Y B} .
$$

\footnotetext{
${ }^{19}$ Note that as shown in $(69)$,

$$
g_{B}=\left[\frac{(1-\alpha-\beta)(\omega+\sigma)}{\beta(\omega+1)}+1\right] g_{K}>g_{K}
$$
}


The first term on the right side of (107) is calculated as follows:

$$
\begin{aligned}
\frac{\lambda \beta Y}{\mu B} & =\frac{\eta_{B} \lambda \beta Y}{v \eta_{Q} Q}=\eta_{B} L_{f}-\frac{(\xi+\zeta) \beta v R C}{Y} \frac{\eta_{B}}{v \eta_{Q} Q} \\
& =\eta_{B} L_{f}-\frac{(\xi+\zeta) \beta v R C}{\mu Y B} .
\end{aligned}
$$

Here, we use (91) for the first and the last equalities, and (90) for the middle equality. From this, (107) is rewritten as

$$
\eta_{B}^{-1} \rho<L_{B}+L_{f}
$$

Combine this with (106), and we have $1>\left(\eta_{B}^{-1}+\eta_{Q}^{-1}\right) \rho$. 